\title{
Full title: Injecting drug use predicts active tuberculosis in a national cohort of people living with HIV from 2000 to 2014
}

Short title: Injecting drug use predicts TB in people with HIV

Joanne R WINTER, MSc, ${ }^{1 *}$ Helen R STAGG, PhD, ${ }^{1}$ Colette J SMITH, PhD, ${ }^{1}$ Alison E BROWN, PhD, ${ }^{2}$

Maeve K LALOR, PhD, ${ }^{2}$ Marc LIPMAN, MD, ${ }^{3,4}$ Anton POZNIAK, MD, ${ }^{5}$ Andrew SKINGSLEY, MRCP, ${ }^{2}$ Peter KIRWAN, BSc, ${ }^{2}$ Zheng YIN, PhD, ${ }^{2}$ H Lucy THOMAS, MFPH, ${ }^{2}$ Valerie DELPECH, $\mathrm{FFPH},{ }^{2 \dagger} \mathrm{Ibrahim}$ ABUBAKAR, $\mathrm{PhD}^{1+}$

1. Institute for Global Health, University College London, 30 Guildford Street, London, WC1N $1 E H, U K$

2. National Infections Service, Public Health England, 61 Colindale Avenue, London, NW9 5EQ, UK

3. Royal Free London National Health Service Foundation Trust, Royal Free Hospital, Pond Street, London, NW3 2QG, UK

4. UCL Respiratory, Division of Medicine, University College London, Royal Free campus, Pond Street, London, NW3 2PF, UK

5. Chelsea and Westminster Hospital, 369 Fulham Road, London, SW10 9NH, UK

* Corresponding author. Telephone: 02076796619. Email: joanne.winter.14@ucl.ac.uk

† Joint senior authors

Word count: 3,386

JRW is funded by a UCL IMPACT studentship. This report is independent research supported by the National Institute for Health Research (Postdoctoral Fellowship, Dr Helen Stagg, PDF-2014-07-008). IA acknowledges funding from NIHR (NF-SI-0616-10037 and SRF-2011-04-001), MRC and the Wellcome Trust. 
This paper utilised two surveillance datasets collected by the respiratory (Tuberculosis section) and HIV departments in the National Infections Service at Public Health England. In light of the work involved in collecting and linking these two datasets, and designing a study utilising both of them, we have listed 13 authors for this paper. 


\section{Abstract and keywords}

\section{Objectives}

3 Tuberculosis (TB) is common in people living with HIV (PLHIV), leading to worse clinical

4 outcomes including increased mortality. We investigated risk factors for developing TB

5 following HIV diagnosis.

\section{Design}

7 Adults aged $\geq 15$ years first presenting to health services for HIV care in England, Wales or

8 Northern Ireland from 2000-2014 were identified from national HIV surveillance data and

9 linked to TB surveillance data.

Methods

11 We calculated incidence rates for TB occurring $>91$ days after HIV diagnosis and investigated

12 risk factors using multivariable Poisson regression.

\section{Results}

1495,003 adults diagnosed with HIV were followed for 635,591 person-years (PY); overall 15 incidence of TB was 344/100,000PY (95\% confidence interval 330-359). TB incidence was high 16 for people who acquired HIV through injecting drugs (PWID; men 876 [696-1,104], women 605 17 [528-593]) and black Africans born in high TB incidence countries (644 [612-677]). The adjusted 18 incidence rate ratio (IRR) for TB amongst PWID was 4.79 [3.35-6.85] for men and 6.18 [3.49-

19 10.93] for women, compared to men who have sex with men. The adjusted IRR for TB in black 20 Africans from high-TB countries was $4 \cdot 27(3 \cdot 42-5 \cdot 33)$, compared to white UK-born individuals.

21 Lower time-updated CD4 count was associated with increased rates of TB.

\section{Conclusions}

23 PWID had the greatest risk of TB; incidence rates were comparable to those in black Africans

24 from high TB incidence countries. Most TB cases in PWID were UK-born, and likely acquired TB [Type text] 
25 through transmission within the UK. Earlier HIV diagnosis and quicker initiation of ART should

26 reduce TB incidence in these populations.

\section{Keywords}

28 HIV, tuberculosis, co-infection, observational study, cohort studies, risk factors

29

30

[Type text] 


\section{Introduction}

Tuberculosis (TB) and HIV are leading causes of morbidity and mortality. Globally, in 2014 there were 1.2 million new cases of TB in people living with HIV (PLHIV), accounting for one in eight TB diagnoses.[1] TB was responsible for one in three HIV-related deaths in 2014.

In England, Wales and Northern Ireland, 25\% of AIDS-defining illnesses from 2001-2010 were TB.[2] The rate of TB disease in PLHIV in the UK was estimated as 328/100,000 person-years (PY) between 1996 and 2005 (excluding patients diagnosed with TB and HIV simultaneously [within 91 days]),[3] and 669/100,000PY across all groups 2007-2011.[4] Estimated TB incidence in the general population is much lower; $10 / 100,000$ population in 2015.[5]

Previous studies in the UK have found higher rates of TB in PLHIV who acquired HIV abroad, or had black African or Indian/Pakistani/Bangladeshi ethnicity, than in white and UK-born populations. $[3,6]$ TB incidence decreased with increasing CD4 count at HIV diagnosis, and was lower for individuals on antiretroviral therapy (ART). However, [6] was limited in its implications for UK TB-HIV control as it was restricted to heterosexuals and did not adjust for time on ART, which is known to be linked to TB incidence.[4] It also included patients diagnosed simultaneously with TB and HIV, many of whom are only diagnosed with HIV as a result of their TB diagnosis.[6] Furthermore, the UK-CHIC study [3] did not provide estimates of TB incidence in PWID.

TB incidence in HIV-positive people who inject drugs (PWID) in the 1980s and 1990s was very high;[7] however the link between TB and HIV-positive PWID in the ART era is less clear. Five cohort studies found TB rates were elevated by a factor of 1.7-4.4 when compared to men who have sex with men (MSM) or people who do not inject drugs,[8-12] whilst one cohort[13] and one cross-sectional study[14] found no significantly increased risk. In the UK, PWID are typically diagnosed with HIV late[15] and have high rates of death,[16, 17] despite good levels of ART coverage (90\%), similar to other risk groups.[18] No recent studies in the UK have

[Type text] 
investigated the risk of TB for PWID. This study aimed to investigate risk factors for developing TB following HIV diagnosis, including HIV acquisition by injecting drug use, to address the paucity of evidence in resource-rich countries in the ART era.

\section{Methods}

\section{Study population}

Adults (aged 15 years or older) notified to Public Health England (PHE)'s HIV and AIDS Reporting System (HARS), first presenting with HIV to health services in England, Wales and Northern Ireland between 2000 and 2014 were included. HARS comprises four linked data sources: reports of all new HIV/AIDS diagnoses and deaths, national laboratory data for CD4 count, annual reporting of demographic and clinical information of PLHIV from all national clinics, and death reports from the Office of National Statistics.[17, 19]

\section{Outcome: TB disease diagnosed from 2000-2014}

TB cases included both culture-confirmed and presumptive (clinical and radiological signs, including a response to specific therapy) diagnoses.

UK HIV and TB surveillance are undertaken separately, necessitating data linkage to analyse co-infection. TB cases across England, Wales and Northern Ireland are reported to the PHE's Enhanced Tuberculosis Surveillance (ETS) system. To identify PLHIV with TB disease, HARS and ETS data were linked using a probabilistic matching algorithm (adapted from [20]), with supplementary deterministic matching to accept/reject borderline matches.[21] Incident TB was defined as TB disease notified to ETS or reported to HARS as a new AIDSdefining illness, that was diagnosed $>91$ days after HIV diagnosis. TB cases diagnosed within 91 days of HIV diagnosis were considered simultaneous diagnoses, to differentiate patients who were not aware of their HIV infection prior to their TB diagnosis. TB cases diagnosed $>91$ days before HIV were considered existing disease. A 91 day threshold for defining simultaneous

[Type text] 
diagnoses was a pragmatic choice to account for delays in diagnosis and reporting, and to exclude ART-induced unmasking immune reconstitution inflammatory syndrome.

\section{Exposure variables}

We included demographic (age at HIV diagnosis, sex, ethnicity, country of birth, TB incidence in country of birth, route of HIV infection, year of HIV diagnosis, index of multiple deprivation [IMD] decile) and clinical (viral load at first presentation, and time-updated CD4 count and ART initiation) exposure variables. IMD score deciles represent relative levels of deprivation of income, employment, health, education, housing and services, crime and living environment for small areas in England and Wales, where 1=most deprived and $10=$ least deprived. $[22,23]$

Composite variables were created combining ethnicity and country of birth or sex and infection route due to mutually exclusive combinations (e.g. being a woman and a MSM is impossible) and known associations. As a proxy TB exposure, countries of birth outside the UK were grouped by TB incidence; 'high incidence' was defined as $>40$ cases $/ 100,000$ adult population in 2013. The most recent IMD data for each country between 2000 and 2014 were used; 2010 for England and 2014 for Wales.

\section{Statistical Analysis}

Data were analysed in Stata version 13.1. Descriptive analyses of the cohort were undertaken. To investigate risk factors for developing TB, we calculated incidence rates of TB per 100,000PY follow-up and assessed TB incidence over time using Nelson-Aalen cumulative hazard plots. We estimated incidence rate ratios using univariable and multivariable Poisson regression models, offset by follow-up time, Cox regression was precluded as our data did not satisfy the proportional hazards assumption for key variables such as route of HIV infection. Individuals diagnosed with TB $\leq 91$ days after HIV diagnosis were excluded to investigate subsequent TB. Follow-up began 92 days from date of HIV diagnosis or first presentation to UK health services and ended on the date of TB diagnosis, death, or 31/12/2014, whichever was earliest. CD4

[Type text] 
count and ART initiation were included as time-updated covariates. Incidence rates for different CD4 strata were calculated using the number of days from each CD4 count to the date of the next CD4 count for each patient. To compare incidence between ART-naïve patients and patients who had initiated ART, we split each patient's follow-up period at the date they first initiated ART to calculate the duration of ART-naïve person-time, and persontime having initiated ART.

Potential confounders and effect modifiers were prospectively identified.[24] Our causal framework determined that viral load should be excluded from the multivariable model because of the potential for causal loops between viral load and CD4 count, which could not be adequately accounted for in the data available. We excluded patients missing data on one or more variables. Linearity (of age, CD4 count and year of HIV diagnosis) and statistical interactions (between ART status and CD4 count) were assessed using likelihood-ratio tests. As we were not investigating a single "main" exposure variable, there were no confounders in the traditional sense, and therefore the multivariable model was informed by a causal inference framework defined a priori. To assess the likely impact of missing data, we compared the distributions of age, sex, route of HIV infection, CD4 count and ethnicity/country of birth for cases with missing vs. complete data on infection route, CD4 count, IMD score and country of birth. Statistical interactions were considered significant at $\mathrm{P}<0.05$. All stated confidence intervals are two-sided $95 \%$ confidence intervals.

Planned sensitivity analyses investigated the impact of using a 6-month threshold (182 days) for simultaneous diagnosis; excluding weaker matches between HARS and ETS; and excluding people who acquired HIV infection through mother-to-child transmission, as the dataset only contained adults and so individuals infected through this route could be missing 15 years follow-up. 


\section{Ethics, consent and permissions}

This analysis was approved by the UCL student Research Ethics Committee (5683/001). PHE has authority under the Health and Social Care Act 2012 to hold and analyse national surveillance data for public health and research purposes.

\section{Role of the funding source}

The funding source had no involvement in the study design; the collection, analysis and interpretation of the data; the writing of the report or the decision to submit the paper for publication.

\section{Results}

\section{Description of co-infected patients}

Between 2000 and 2014, 102,202 adults were newly diagnosed with HIV, among whom 5,649 (6\%) had TB. 3,103 (55\%) were simultaneously diagnosed with TB and HIV, 2,187 (39\%) developed TB after $>91$ days and $359(6 \%)$ were diagnosed with TB first (Table 1).

Of people with TB who acquired HIV infection through heterosexual sex, over half were diagnosed simultaneously with TB and HIV; $60 \%$ for men and $54 \%$ for women. In contrast, more TB cases in MSM and PWID were diagnosed more than 91 days after diagnosis of HIV infection ( $51 \%$ and $54 \%$, respectively). The proportion of TB cases occurring after HIV diagnosis was highest in white, UK-born individuals $(179 / 359,48 \%)$ and those born in low TB incidence countries $(116 / 245,47 \%)$; these two groups comprise $38 \%$ of the cohort.

\section{Incidence of TB following HIV diagnosis}

95,003 adults were TB-free 92 days after presenting for HIV care, with a total of 635,591PY follow-up. Median age at HIV diagnosis was 34 years (inter-quartile range [IQR] 28-42) and median CD4 count was 340 cells/ $\mu$ I (IQR 170-527). 95\% of patients had $>1$ CD4 count (median 14).

[Type text] 
153 Overall TB incidence was 344/100,000PY (95\% Cl: 330-359, Table 1). The probability of

154 developing TB was highest in the year following HIV diagnosis and then decreased (Figure 1a).

155 Incidence was high in PWID (men 876/100,000 [696-1,104/100,000]; women 605/100,000

$156[386-949 / 100,000])$ and heterosexuals (men 598/100,000 [555-645/100,000], women 559

157 [528-593/100,000]), particularly compared with MSM (111/100,000 [98-126/100,000]). The

158 largest differences in cumulative probability of TB diagnosis between PWID, black Africans

159 from high-TB incidence countries and MSM were in the first two years following HIV diagnosis;

160 the rate of diagnosis remained relatively constant across all groups thereafter (Figure 1b).

161 TB incidence increased with decreasing time-updated CD4 count, from 139/100,000 (123-

$162157 / 100,000)$ for those with CD4 count $\geq 500$ cells/ $\mu$ l to $2,788 / 100,000(2,368-3,282 / 100,000)$

163 for those with CD4 count $<50$ cells $/ \mu$ l. TB incidence was $511 / 100,000(484-539 / 100,000)$ in

164 people who had never received ART (26\% of all PY) compared to $228 / 100,000$ (213-

$165243 / 100,000)$ in people who had $(74 \%$ of PY). TB incidence was higher for PWID who had never

166 initiated ART $(1,478 / 100,000$ [95\% Cl 1,157-1,888/100,000] than for black Africans from high-

167 TB incidence countries who had never initiated ART (991/100,000 [929-1,058/100,000])

168 although incidence rates following ART initiation were similar in both groups $(384 / 100,000$

169 [264-560/100,000] for PWID versus $421[389-456 / 100,000]$ for black Africans). TB incidence

170 was highest in those living in areas of England and Wales with the lowest decile of IMD score

$171(485 / 100,000[437-537 / 100,000])$.

\section{Factors associated with developing TB disease}

17362,684 individuals with complete case data and a TB-free follow-up period of $>91$ days

174 following HIV diagnosis were included in the time-to-event analysis. There were a total of

175414,714 PY of follow-up (median follow-up 7·1 years, IQR 3.6-10.4), during which there were

176 1,591 TB diagnoses (Table 2). The median duration of follow-up was 7.3 years (IQR 3.9-10.4)

177 for patients who did not develop TB, whilst patients who did develop TB did so in a median of

[Type text] 
0.2 years (IQR 0.1-0.5). Black African patients born in high-TB countries had a slightly higher median follow-up period of 8.2 (4.7-11.0) years, compared to 6.3 (3.1-9.8) for MSM and 6.5 (3.4-9.8) for PWID, as black Africans were more likely to be diagnosed earlier in the study period than PWID or MSM.

All exposures were included in the multivariable Poisson regression model (Table 2), except viral load and IMD decile. IMD decile was excluded as there was a high degree of missing data and no association with the outcome in a multivariable model (supplementary tables 1-3). CD4 count and age at HIV diagnosis were treated as categorical variables (tests for linearity $\mathrm{P}<0.001, \mathrm{P}=0.005$, respectively), year of HIV diagnosis was treated as a linear variable $(P>0.05)$.There was a statistically significant interaction between time-updated CD4 count and time-updated ART status $(\mathrm{P}<0 \cdot 001)$.

Compared to MSM, PWID had increased rates of TB (incidence rate ratio [IRR] for men $5 \cdot 47$

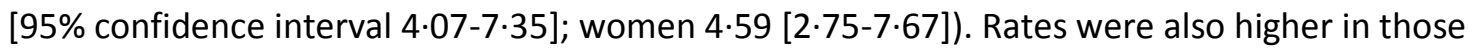
infected through heterosexual sex (men $1 \cdot 70$ [1.38-2.10]; women 1.86 [1.51-2.29]). UK-born black Africans (1.97 [1.10-3.51]) and people of other ethnicities (1.92 [1.29-2.84]) were associated with increased incidence rates versus white UK-born individuals, as were those born in high TB incidence countries (black African 4.27 [3·42-5·33], white 2·19 [1·53-3·15], other ethnicities $3 \cdot 36$ [2·57-4·39]).

Overall, and within each stratum of CD4 count, TB rates were greatly reduced in individuals who had received ART compared to those who had not (Table 3). When stratifying by ART initiation status, lower time-updated CD4 count was strongly associated with increased TB rates (Table 4). For individuals who had never initiated ART, the IRR for TB increased with decreasing CD4 count to $6 \cdot 42[4 \cdot 87-8 \cdot 46]$ for $0-49$ cells $/ \mu \mathrm{l} c f . \geq 500$ cells $/ \mu$ l. The increased risk at low CD4 count was higher in individuals who had initiated ART, with an IRR of $44 \cdot 21$ [30.9063.24] for 0-49 cells/ $\mu \mathrm{l}, c f . \geq 500$ cells $/ \mu \mathrm{l}$.

[Type text] 
In a post-hoc analysis of patients who had initiated ART, we found that those who developed TB were more likely to have discontinued ART at their last clinic visit $(27 \%$, versus $6 \%$ of those without $T B, P<0.001$, Supplementary table 4). ART initiation rates and time from the most recent clinic visit to the end of the study were similar for MSM, heterosexuals and PWID.

There was no substantial difference in the age, sex, ethnicity/country of birth, route of HIV infection or CD4 count of patients with missing data on any of the following variables: route of HIV infection, CD4 count, IMD decile and country of birth. Patients with missing route of infection were less likely to be diagnosed with TB; however there were no substantial differences for patients missing data on any other variable.

\section{Sensitivity analysis}

Sensitivity analyses were conducted as follows: (1) excluding 241 individuals who acquired HIV infection through mother-to-child transmission, (2) excluding 595 individuals with TB whose probabilistic matching scores (linking to their HIV record) were in the lowest quartile, (3) excluding 137 individuals with TB who were matched to their HIV record using the three lowest-ranked deterministic criteria, (4) excluding 424 individuals diagnosed with TB 92-182 days after HIV diagnosis, (5) including IMD score and excluding data on 12,432 individuals missing IMD score. All analyses provided consistent results with the main model (supplementary tables 1-3).

\section{Discussion}

People who acquired HIV infection through injecting drug use (largely UK-born patients) had a high risk of TB following their HIV diagnosis, with incidence rates comparable to those in black Africans born in high TB incidence countries; almost five-fold more than MSM after accounting for other factors including starting ART. Consistent with previous research, $[3,6]$ declining CD4 count was associated with higher TB rates. 
This study benefits from the very large national HIV-positive cohort, providing comprehensive results for England, Wales and Northern Ireland. The algorithm linking patients with TB and HIV utilises ethnicity, year and country of birth; all variables with very high completeness: $97 \cdot 3 \%, 99 \cdot 9 \%$ and $90 \cdot 5 \%$ respectively.

We found no substantial differences in the demographics or proportion of TB in patients missing data on each of these variables; however patients missing data on one variable were more likely to have other missing data. Additionally, patients missing data for multiple variables were less likely to be linked to a TB notification and therefore we may have underestimated TB incidence rates; it is likely that the low incidence of TB in patients with "unknown" route of HIV infection is a symptom of this and patients with extensive missing data may be more likely to be from populations at high risk for TB. Additionally, the record linkage algorithm is less sensitive to non-English names,[20] therefore we may have underestimated TB incidence in foreign populations.

One limitation was missing CD4 count data for approximately a third of patients, who were therefore excluded from the risk factor analysis. This is partly due to difficulties linking data, and partly because some large hospitals do not supply CD4 count data to HARS. However, we found no evidence that patients with missing CD4 count data were systematically different to our analysis cohort. As our sample size remained very large, and there was no evidence that patients missing data were systematically different, we chose not to use multiple imputation due to the complexity of the dataset as a result of using time-updated CD4 count and ART initiation. Data were available on ART discontinuation, but were of poor quality and could not be included in the model. Consequently we may have underestimated the association between starting ART and lower TB incidence by assuming all individuals remained on treatment for the duration of our study. 
Individuals entered the study cohort 92 days after HIV diagnosis or first presentation to UK health services; therefore we may have underestimated TB incidence in people diagnosed abroad who were at risk prior to entering the UK, as we would have missed TB cases diagnosed during the initial period following HIV diagnosis when TB incidence is highest. A recent study of PLHIV had $18 \%$ loss to follow-up over 4 years, and $14 \%$ of TB cases diagnosed $>91$ days after HIV diagnosis were in these patients.[4] As TB and HIV are sometimes treated (and usually reported) separately in the UK, dropping out of HIV care does not prevent notification of a TB diagnosis. We therefore used passive censoring, continuing follow-up until 31/12/2014 rather than the date last seen for HIV care. Consequently, migration out of the UK may mean we underestimated TB incidence.

A limitation of the Poisson regression model was censoring due to competing risks, specifically deaths from non-TB causes. However, few patients died (3\%) and median time to death was 3.4 years, substantially longer than median time to TB diagnosis ( 1.8 years); therefore any impact of censoring is likely to be minimal.

While PWID represented $<2 \%$ of PLHIV, they accounted for $3 \%$ of TB cases in this population and $>4 \%$ of cases diagnosed $>91$ days after HIV diagnosis. TB incidence in PWID in our study (876/100,000PY in men and 605/100,000 in women) was substantially higher than that in a cohort of German PLHIV,[25] possibly because this cohort utilises active rather than passive follow-up and excluded patients who did not present to care for 6 months or more, who may be more likely to develop TB disease than patients who remain engaged with care. PWID are typically diagnosed with HIV late,[18] have slower rates of linkage to care and lower rates of viral suppression,[26] all of which may contribute to increased risk of TB. We found ART initiation and the time from the last clinic visit to the end of the study were comparable for MSM, heterosexuals and PWID; and that PWID did not have higher rates of ART discontinuation at their last clinic visit prior to study end (Supplementary Table 4). 
276 Consequently, it seems high rates of TB among PWID are caused by difficulties in linking to

277 care and not lack of engagement with health services once linked. Many PWID have other co-

278 morbidities which may cause immunosuppression, make HIV care more challenging, or be

279 associated with increased risk of TB.[27] Additionally there are high rates of alcoholism and

280 homelessness, and living in hostels is common.[28] These, in addition to injecting drugs in

281 shared social settings, may drive close mixing of people with similar risk factors for TB disease,

282 driving transmission. High rates of smoking may also have impacts on both local lung immunity

283 and TB transmission. Further studies are needed to explore the impact of these factors and to

284 design effective interventions. BHIVA guidelines currently recommend testing and treating

285 LTBI among PLHIV using criteria based on CD4 count, time on ART and country of birth.[29] As

286 the incidence of TB among PWID was comparable to that of black African patients born in

287 countries with high TB incidence, we suggest that additionally screening and treating PWID for

288 LTBI should be considered.

The majority of PWID were white (51\%) and born in the UK or low TB incidence countries

290 (72\%). It is therefore likely that most TB in this group was acquired in the UK, meaning these cases may be preventable by diagnosing HIV sooner and ensuring prompt ART initiation. We could also do more to diagnose TB cases sooner; the impact of active case finding in PLHIV should be evaluated. In contrast, heterosexuals were typically black African (61\%) and born in high TB incidence countries (69\%), both populations which also have high rates of TB among HIV-negative people. Consequently, they are likely to have acquired TB abroad, limiting our ability to prevent these TB infections if they present with clinical TB at the time of HIV diagnosis.[30] As $>60 \%$ of heterosexuals were diagnosed with TB simultaneously or prior to HIV diagnosis, greater efforts to diagnose these HIV infections and initiate ART would reduce TB in this population. A greater focus on screening and treating latent TB infection (LTBI) could also prevent these cases.[31] There is little data available on the prevalence of LTBI and the 
preventive therapy vary substantially between HIV clinics,[32, 33] and a survey of UK HIV healthcare providers providing care to $90 \%$ of PLHIV in the UK found that only $54 \%$ offered LTBI screening and preventive therapy.[34] Health economics evaluations would be useful to determine the most effective screening measures for these populations.

Over half of all TB cases (55\%) were diagnosed simultaneously with HIV infection, and of the $39 \%$ diagnosed later, the probability of a TB diagnosis was highest in the first year following HIV diagnosis (Figure 1). This suggests that TB disease is largely the result of TB infection acquired prior to HIV diagnosis. This could result from late diagnosis of existing active TB, particularly in migrants who have recently moved to the UK from high-burden countries and whose TB is largely attributable to reactivation of remotely acquired infection.[35] Additionally, the incidence of TB amongst migrants decreases with time since entry to the UK, as new TB infection is less likely in the UK than their country of origin. Other factors which could explain this trend are increased surveillance for opportunistic infections following HIV diagnosis, or "unmasking-type" immune reconstitution inflammatory syndrome as a consequence of ART. Whilst TB incidence was lower after the first year since HIV diagnosis (Table 1), $25 \%$ of all TB cases occurred more than one year after HIV diagnosis. These cases can certainly be attributed to reactivation of LTBI and could be preventable with LTBI treatment.

Patients who had initiated ART had greatly reduced rates of TB compared to those who had not (Table 3); however time-updated CD4 count and ART initiation status interacted within our model. Higher rate ratios for TB at low CD4 count in people on ART may be attributable to late ART start (i.e. long periods of low CD4 count prior to initiating ART and then little time on ART prior to TB diagnosis), or due to ART discontinuation. The SMART trial demonstrated an association between stopping ART and increased risk of opportunistic disease and death.[36] Our post-hoc analysis of patients who had started ART demonstrated that patients who went on to develop TB were more likely to have discontinued ART at their last study visit than 
individuals who remained TB-free (Table 5). This suggests ART discontinuation could leave patients at risk of new TB disease.

In England, Wales and Northern Ireland, PLHIV who acquired HIV by injecting drugs had higher rates of TB after their HIV diagnosis than MSM, comparable to black Africans born in countries with high TB incidence. High rates of TB in PWID are likely to result from transmission within the UK. ART is highly protective against TB, but the majority of TB diagnoses were in people who have never started ART. ART discontinuation rates were much higher in people who subsequently developed TB than those who did not. Quicker initiation of ART, as per the recently updated BHIVA guidelines,[37] and improving retention in care and ART continuation should decrease incident TB in PLHIV.

\section{Acknowledgements}

JRW is funded by a UCL IMPACT studentship. This report is independent research supported by the National Institute for Health Research (Postdoctoral Fellowship, Dr Helen Stagg, PDF-201407-008). IA acknowledges funding from NIHR (NF-SI-0616-10037 and SRF-2011-04-001), MRC and the Wellcome Trust. The views expressed in this publication are those of the authors and not necessarily those of the NHS, the National Institute for Health Research or the Department of Health.

\section{Author contributions}

JRW designed the study, linked the TB and HIV surveillance datasets, conducted the analysis and drafted the paper. HRS and CS designed the study, analysed and interpreted the data and critically revised the paper. $A B, M K L, A S, H L T, Z Y$ and $P K$ gave input on the study design, collected the data, linked the datasets, interpreted the results and critically revised the paper. VD and IA designed the study, collected, linked, analysed and interpreted the data and revised the paper. ML and AP interpreted the results and critically revised the paper. All authors approved the final version of the paper for publication.

[Type text] 
352 Declaration of interests

353 JRW, AEB, MKL, ML, AS, PK, ZY, HLT, VD and IA have no conflicts of interests to declare. HRS

354 declares funding from the National Institute for Health Research, UK during the conduct of the

355 study; and, outside of the submitted work, grants and personal fees from Otsuka

356 Pharmaceutical, non-financial support from Sanofi, and other support from the WHO. Outside

357 the submitted work, CJS reports personal fees from Gilead Sciences and ViiV Healthcare. AP is

358 chair of the BHIVA TB guidelines committee. JRW had full access to all the data in the study

359 and had final responsibility for the decision to submit for publication.

360

[Type text] 
362 1. WHO. Tuberculosis and HIV 2016. [Accessed 01/07/2016]. Available from:

363 http://www.who.int/hiv/topics/tb/about_tb/en/.

$3642 . \quad H e a l$ th Protection Agency. HIV in the United Kingdom: 2011 report. London: Health

365 Protection Services, Colindale. 2011.

$3663 . \quad$ United Kingdom Collaborative HIV Cohort Study Group, Grant AD, Bansi L, Ainsworth J, 367 Anderson J, Delpech V, et al. Tuberculosis among people with HIV infection in the United 368 Kingdom: opportunities for prevention? Aids. 2009;23(18):2507-15.

369 4. Gupta RK, Rice B, Brown AE, Thomas HL, Zenner D, Anderson L, et al. Does antiretroviral therapy reduce HIV-associated tuberculosis incidence to background rates? A national observational cohort study from England, Wales, and Northern Ireland. The lancet HIV. 2015;2(6):e243-51.

5. WHO. WHO TB burden estimates 2017. [Accessed 23/07/2017]. Available from: http://www.who.int/tb/country/data/download/en/.

6. Rice B, Elford J, Yin Z, Kruijshaar M, Abubakar I, Lipman M, et al. Decreasing incidence of tuberculosis among heterosexuals living with diagnosed HIV in England and Wales. Aids. 2013;27(7):1151-7.

7. Selwyn PA, Hartel D, Lewis VA, Schoenbaum EE, Vermund SH, Klein RS, et al. A prospective study of the risk of tuberculosis among intravenous drug users with human immunodeficiency virus infection. New England Journal of Medicine. 1989;320(9):545-50. 8. Monge S, Diez M, Pulido F, Iribarren JA, Campins AA, Arazo P, et al. Tuberculosis in a cohort of HIV-positive patients: epidemiology, clinical practice and treatment outcomes. The international journal of tuberculosis and lung disease : the official journal of the International Union against Tuberculosis and Lung Disease. 2014;18(6):700-8.

9. Girardi E, Sabin CA, d'Arminio Monforte A, Hogg B, Phillips AN, Gill MJ, et al. Incidence of Tuberculosis among HIV-infected patients receiving highly active antiretroviral therapy in Europe and North America. Clin Infect Dis. 2005;41(12):1772-82.

10. Sterling TR, Lau B, Zhang J, Freeman A, Bosch RJ, Brooks JT, et al. Risk factors for tuberculosis after highly active antiretroviral therapy initiation in the United States and Canada: implications for tuberculosis screening. The Journal of infectious diseases. 2011;204(6):893-901.

11. Taarnhoj GA, Engsig FN, Ravn P, Johansen IS, Larsen CS, Roge B, et al. Incidence, risk factors and mortality of tuberculosis in Danish HIV patients 1995-2007. BMC pulmonary medicine. 2011;11:26.

12. Mor Z, Lidji M, Cedar N, Grotto I, Chemtob D. Tuberculosis incidence in HIV/AIDS patients in Israel, 1983-2010. PloS one. 2013;8(11):e79691.

13. Moreno S, Jarrin I, Iribarren JA, Perez-Elias MJ, Viciana P, Parra-Ruiz J, et al. Incidence and risk factors for tuberculosis in HIV-positive subjects by HAART status. The international journal of tuberculosis and lung disease : the official journal of the International Union against Tuberculosis and Lung Disease. 2008;12(12):1393-400.

14. Zhang $Y, Y u L$, Tang ZR, Huang SB, Zheng YJ, Meng ZH, et al. Diagnosis of pulmonary tuberculosis among asymptomatic HIV+ patients in Guangxi, China. Chinese medical journal. 2010;123(23):3400-5.

15. Skingsley A, Yin Z, Kirwan P, Croxford S, Chau C, Conti S, et al. HIV in the UK - Situation report 2015: Data to end 2014. Public Health England, London: 2015.

16. Yin Z, Brown AE, Hughes G, Nardone A, Gill ON, Delpech VC, et al. HIV in the United Kingdom 2014 report: data to end 2013. Public Health England, London. 2014.

17. Zenner D, Abubakar I, Conti S, Gupta RK, Yin Z, Kall M, et al. Impact of TB on the survival of people living with HIV infection in England, Wales and Northern Ireland. Thorax. 2015;70(6):566-73.

[Type text] 
411 18. Skingsley A, Kirwan P, Yin Z, Nardone A, Hughes G, Tosswill J, et al. HIV new

412 diagnosies, treatment and care in the UK 2015 report: Data to end 2014. Public Health

413 England, London: 2015.

414 19. Gupta RK, Brown AE, Zenner D, Rice B, Yin Z, Thomas HL, et al. CD4+ cell count 415 responses to antiretroviral therapy are not impaired in HIV-infected individuals with 416 tuberculosis co-infection. Aids. 2015;29(11):1363-8.

417 20. Aldridge RW, Shaji K, Hayward AC, Abubakar I. Accuracy of Probabilistic Linkage Using 418 the Enhanced Matching System for Public Health and Epidemiological Studies. PloS one. 419 2015;10(8).

420 21. Winter JR, Delpech V, Kirwan P, Stagg HR, Venugopalan S, Skingsley A, et al. Linkage of 421 UK HIV and Tuberculosis Data Using Probabilistic and Deterministic Methods. Conference on 422 Retroviruses and Opportunistic Infections; Boston, USA 2016.

423 22. Department for Communities and Local Government. English Indices of Deprivation

424 2015. [Accessed 20/05/2016]. Available from:

425 https://www.gov.uk/government/collections/english-indices-of-deprivation.

426 23. Welsh Govenment. Welsh Index of Multiple Deprivation (WIMD) 2015. [Accessed

427 20/05/2016]. Available from: http://gov.wales/statistics-and-research/welsh-index-multiple-

428 deprivation/?lang=en.

429 24. Victora CG, Huttly SR, Fuchs SC, Olinto MTA. The role of conceptual frameworks in 430 epidemiological analysis: A hierarchical approach. International Journal of Epidemiology. 431 1997;26(1):224-7.

432 25. Karo B, Haas W, Kollan C, Gunsenheimer-Bartmeyer B, Hamouda O, Fiebig L, et al. 433 Tuberculosis among people living with HIV/AIDS in the German ClinSurv HIV Cohort: long434 term incidence and risk factors. BMC infectious diseases. 2014;14:148.

435 26. Kirwan PD, Chau C, Brown AE, Gill ON, Delpech VC, Contributors. HIV in the UK - 2016 436 report. Public Health England, London. 2016.

437 27. Public Health England, Health Protection Scotland, Public Health Wales, Public Health 438 Agency Northern Ireland. Shooting Up: Infections among people who inject drugs in the UK, 439 2015. London: Public Health England, 2016 November 2016. Report No.

440 28. National AIDS Trust. HIV and injecting drug use. London: 2013.

441 29. Pozniak AL, Coyne KM, Miller RF, Lipman MCI, Freedman AR, Ormerod LP, et al. British 442 HIV Association guidelines for the treatment of TB/HIV coinfection 2011. HIV Medicine. 443 2011;12:517-24.

444 30. Rice BD, Elford J, Yin Z, Delpech VC. A new method to assign country of HIV infection 445 among heterosexuals born abroad and diagnosed with HIV. Aids. 2012;26(15):1961-6.

446 31. Temprano ANRS Study Group, Danel C, Moh R, Gabillard D, Badje A, Le Carrou J, et al. 447 A Trial of Early Antiretrovirals and Isoniazid Preventive Therapy in Africa. The New England 448 journal of medicine. 2015;373(9):808-22.

449 32. Fox-Lewis A, Brima N, Muniina P, Grant AD, Edwards SG, Miller RF, et al. Tuberculosis 450 screening in patients with HIV: An audit against UK national guidelines to assess current practice and the effectiveness of an electronic tuberculosis-screening prompt. Int J STD AIDS. 2016;27(10):901-5.

33. Kall MM, Coyne KM, Garrett NJ, Boyd AE, Ashcroft AT, Reeves I, et al. Latent and subclinical tuberculosis in HIV infected patients: a cross-sectional study. BMC infectious diseases. 2012;12:107.

34. White HA, Miller RF, Pozniak AL, Lipman MC, Stephenson I, Wiselka MJ, et al. Latent tuberculosis infection screening and treatment in HIV: insights from evaluation of UK practice. Thorax. 2017;72(2):180-2.

35. Aldridge RW, Zenner D, White PJ, Williamson EJ, Muzyamba MC, Dhavan P, et al. Tuberculosis in migrants moving from high-incidence to low-incidence countries: a 
461 population-based cohort study of 519955 migrants screened before entry to England, Wales, 462 and Northern Ireland. Lancet (London, England). 2016;388(10059):2510-8.

463 36. Strategies for Management of Antiretroviral Therapy Study G, Lundgren JD, Babiker A, 464 El-Sadr W, Emery S, Grund B, et al. Inferior clinical outcome of the CD4+ cell count-guided 465 antiretroviral treatment interruption strategy in the SMART study: role of CD4+ Cell counts 466 and HIV RNA levels during follow-up. The Journal of infectious diseases. 2008;197(8):1145-55. $46737 . \quad B H I V A$. British HIV Association guidelines for the treatment of HIV-1-positive adults 468 with antiretroviral therapy 2015. London: 2015. 


\section{$471 \quad$ Figures}

472 Figure 1: Cumulative hazard plot of the probability of developing TB from $>91$ days following 473 HIV diagnosis

474

[Type text] 


\section{Tables}

476 Table 1: TB diagnoses in people notified with HIV from 2000 to 2014 in England, Wales and Northern Ireland, and the incidence rates of TB in people who

477 were diagnosed with TB >91 days following HIV diagnosis.

\begin{tabular}{|c|c|c|c|c|c|c|c|c|}
\hline & HIV cases & TB cases & & & & & & \\
\hline & & Total & $\begin{array}{l}\text { Prior to HIV } \\
\text { diagnosis }\end{array}$ & $\begin{array}{l}\text { Simultaneous with } \\
\text { HIV diagnosis }\end{array}$ & Following $\mathrm{HI}$ & diagnosis & & \\
\hline & n (column \%) & n (row \%) & n (row \%) & n (row \%) & n (row \%) & PY follow-up & Incidence rate* $(95 \% \mathrm{Cl})$ & $\begin{array}{l}1 \text { year from HIV } \\
\text { diagnosis* }(95 \% \mathrm{Cl})\end{array}$ \\
\hline Total & 102,202 & $5,649(5.5)$ & $359(6)$ & $3,103(55)$ & 2,187 (39) & 635,591 & $344(330-359)$ & $247(234-260)$ \\
\hline Route & & & & & & & & \\
\hline MSM & $35,879(35.1)$ & 462 (1.3) & $31(7)$ & $195(42)$ & $236(51)$ & 212,844 & $111(98-126)$ & $86(74-100)$ \\
\hline
\end{tabular}




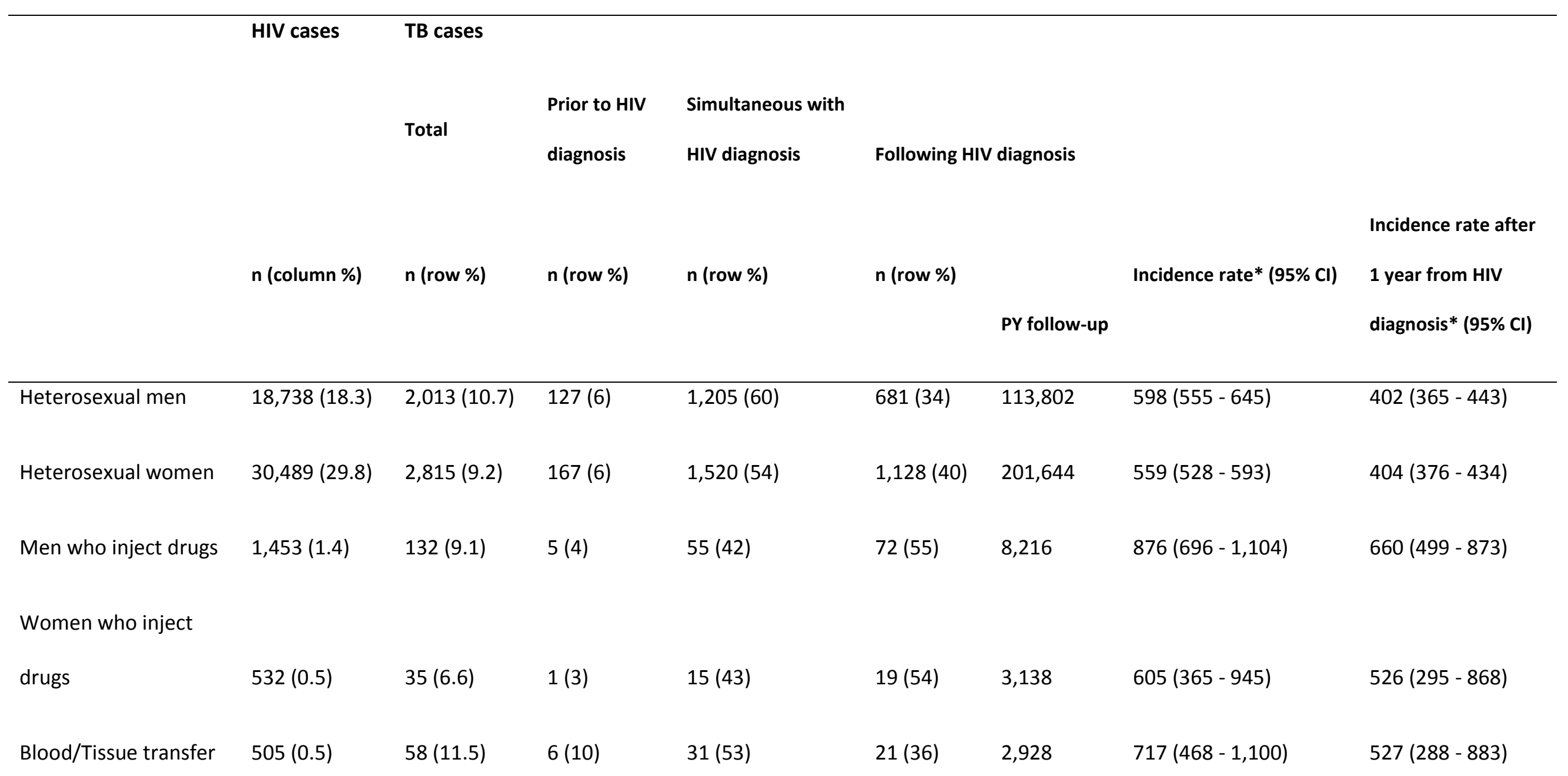




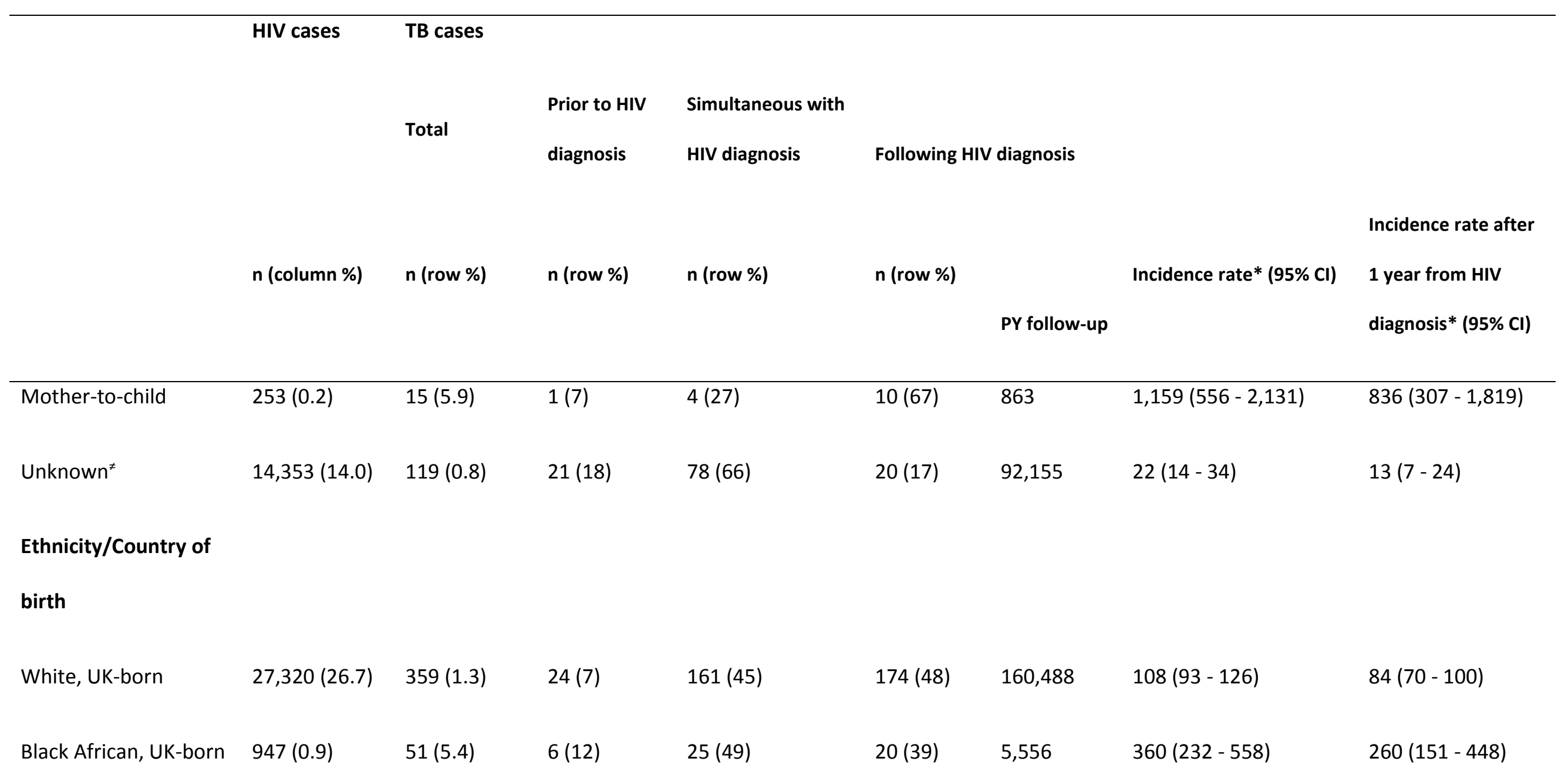




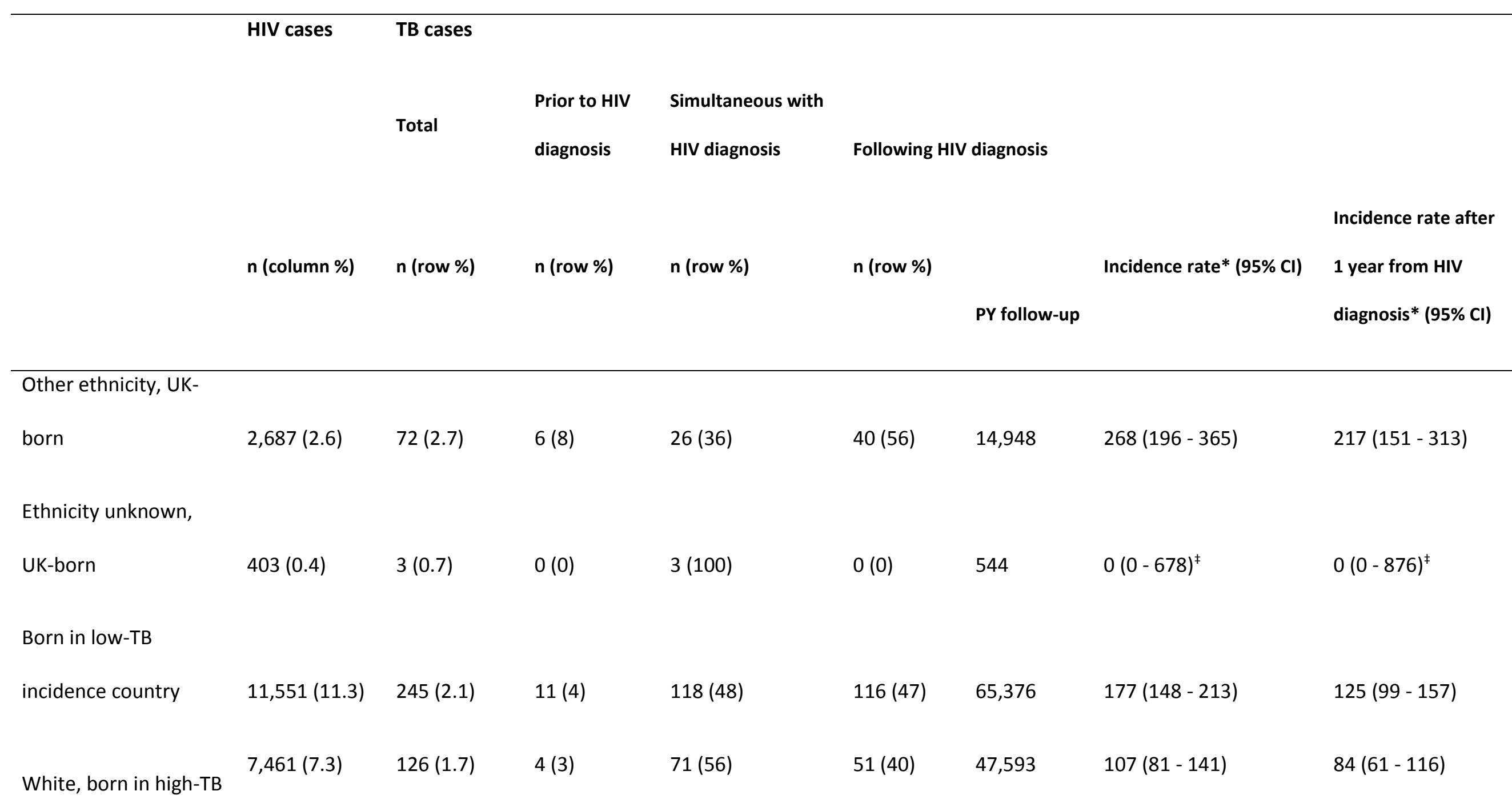




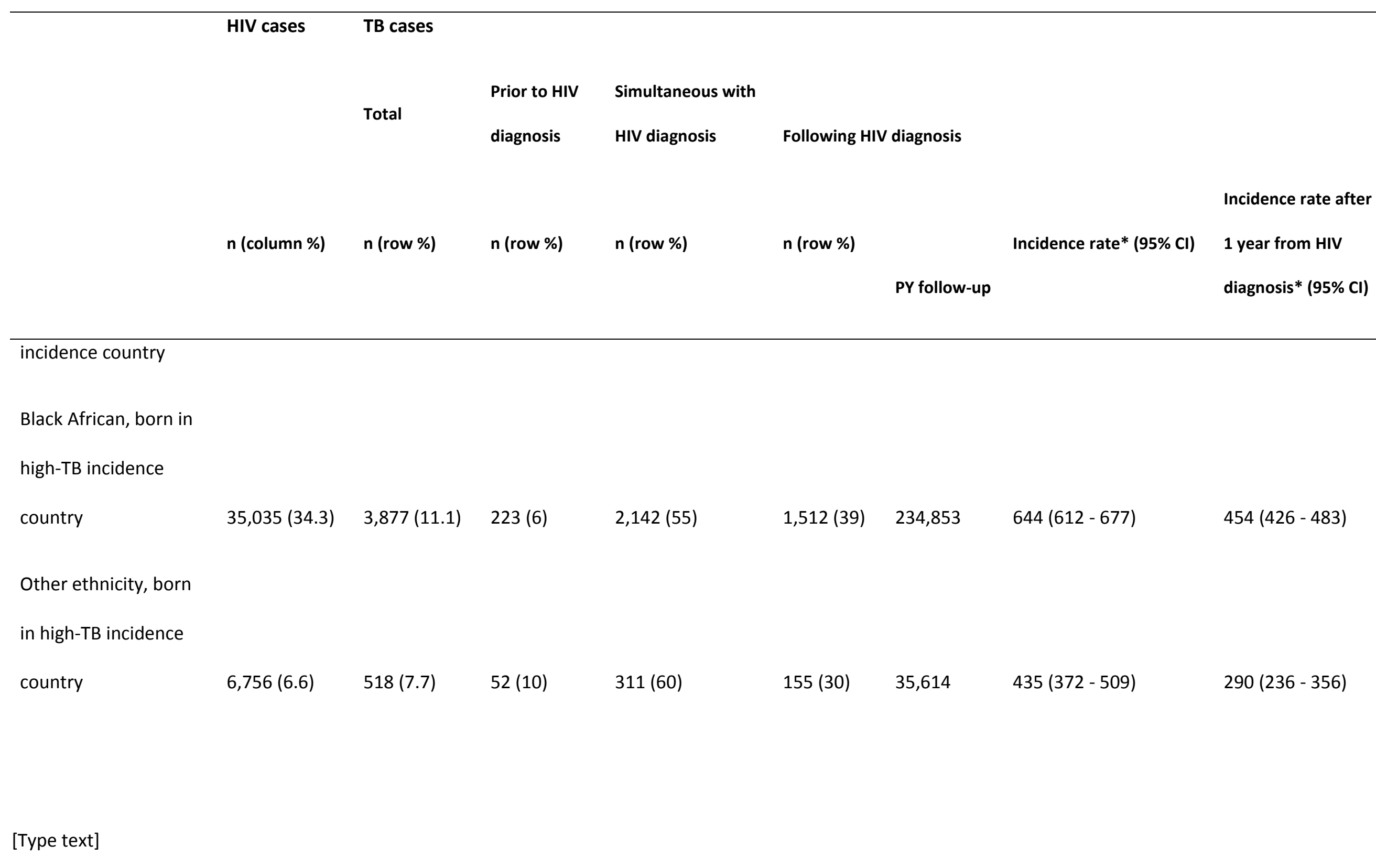




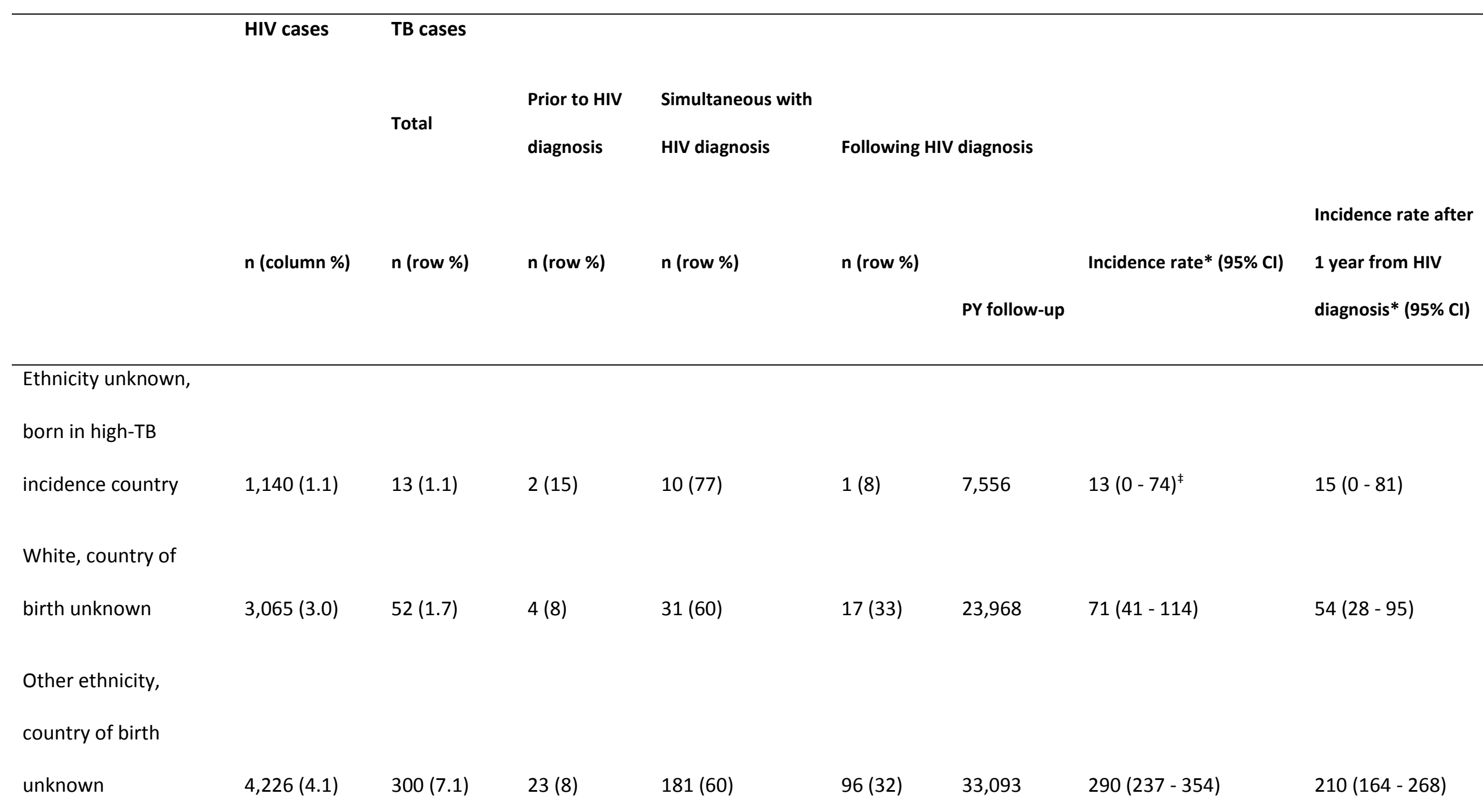

[Type text] 


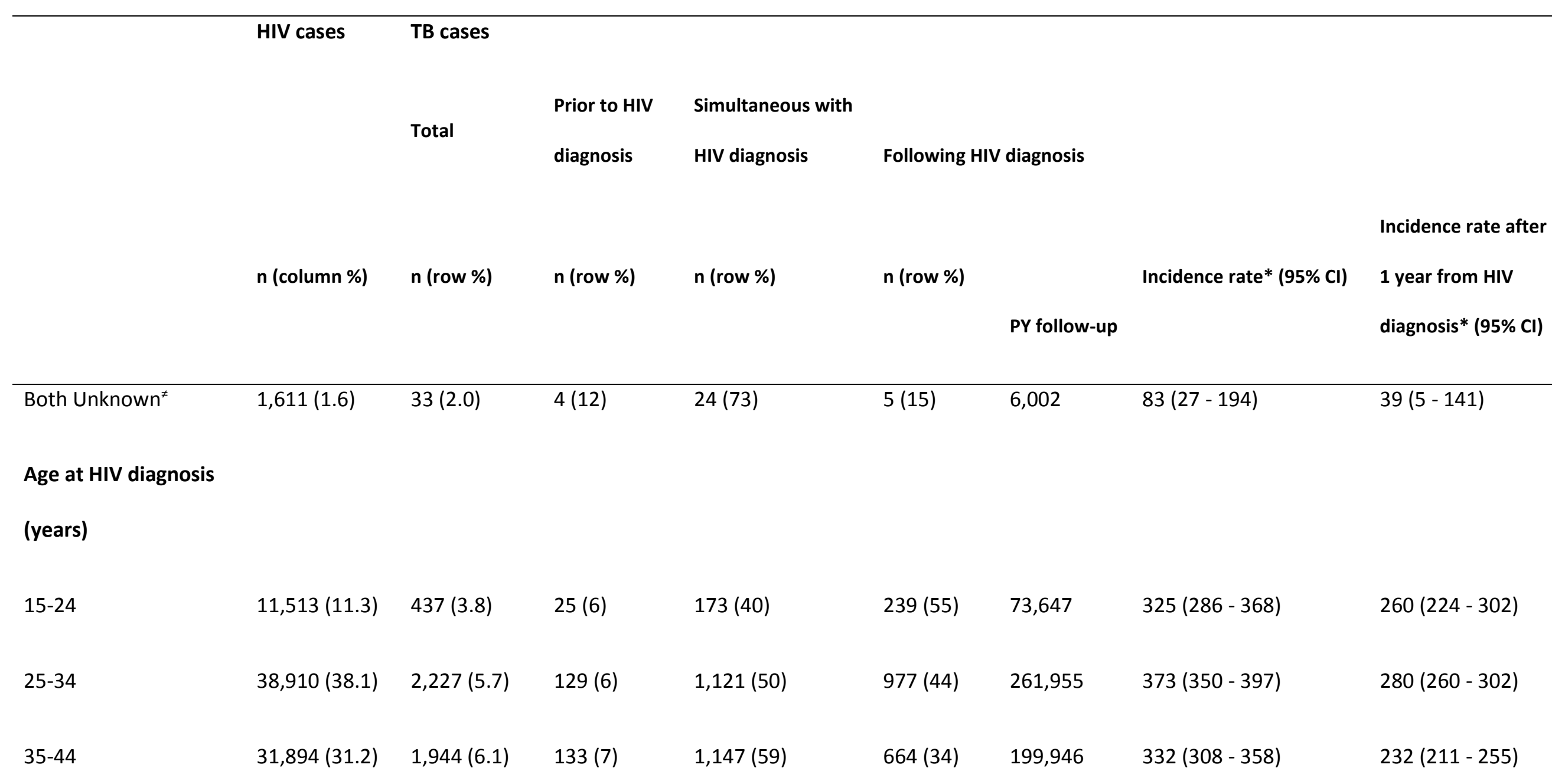




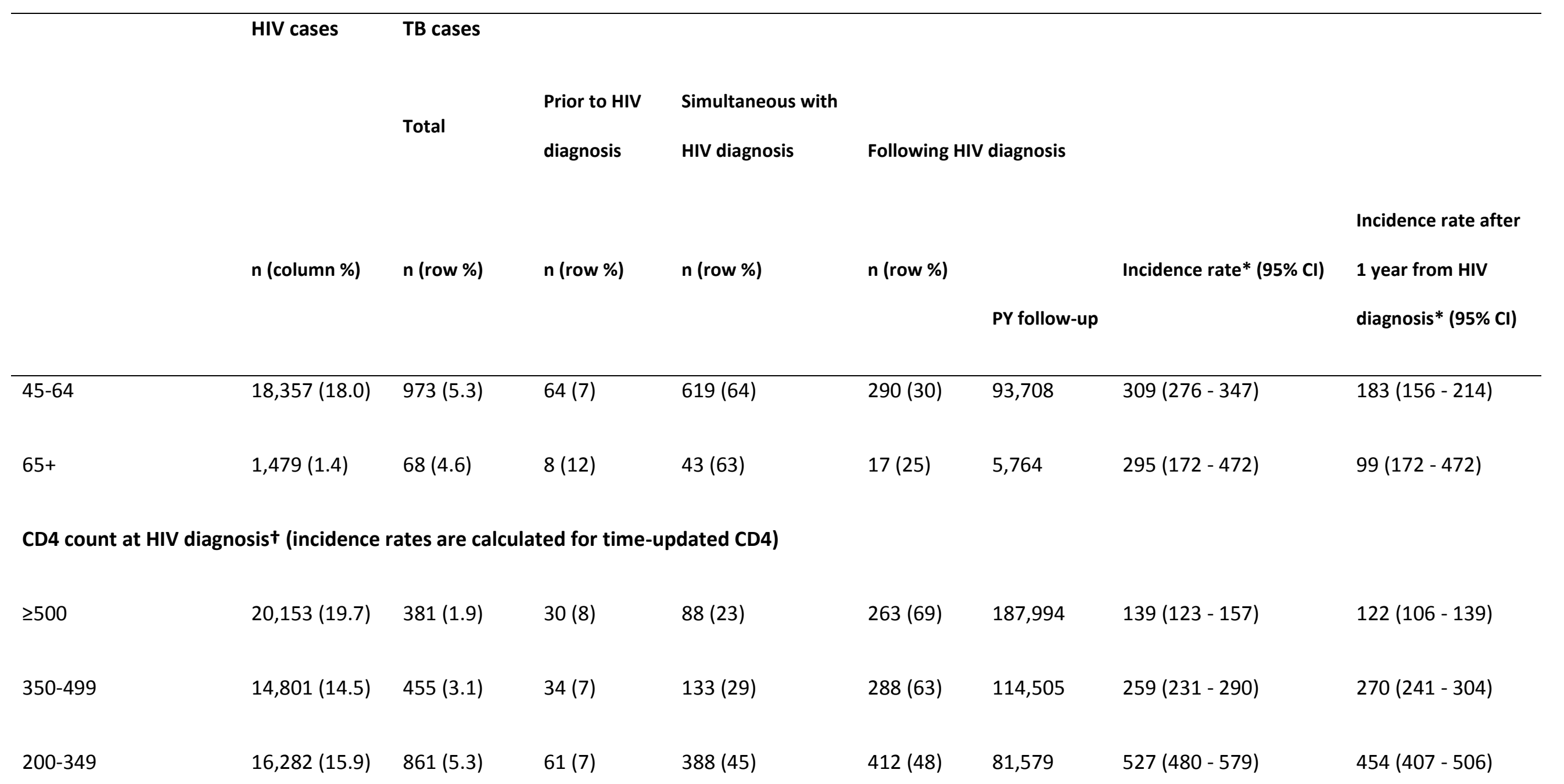




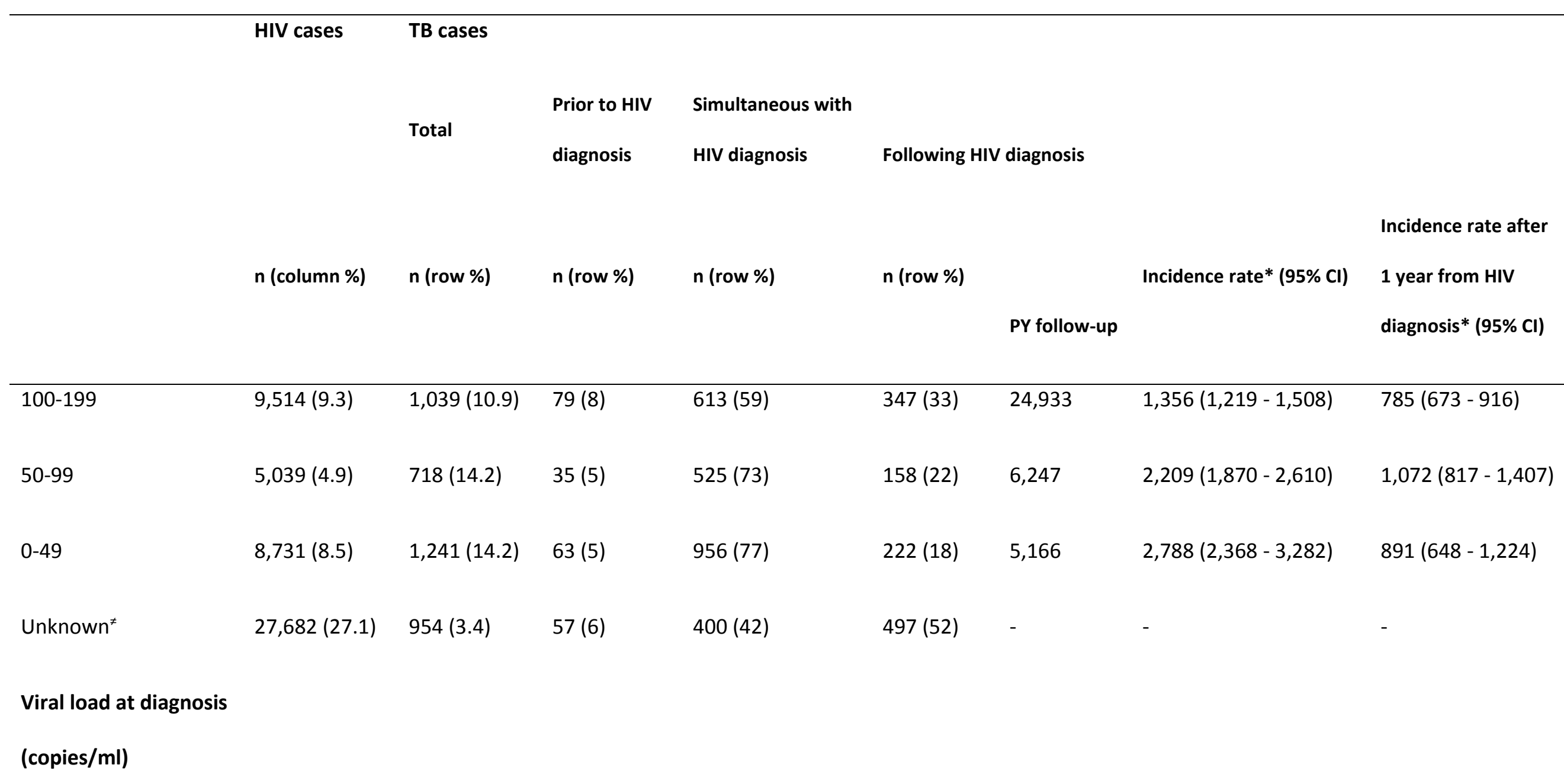




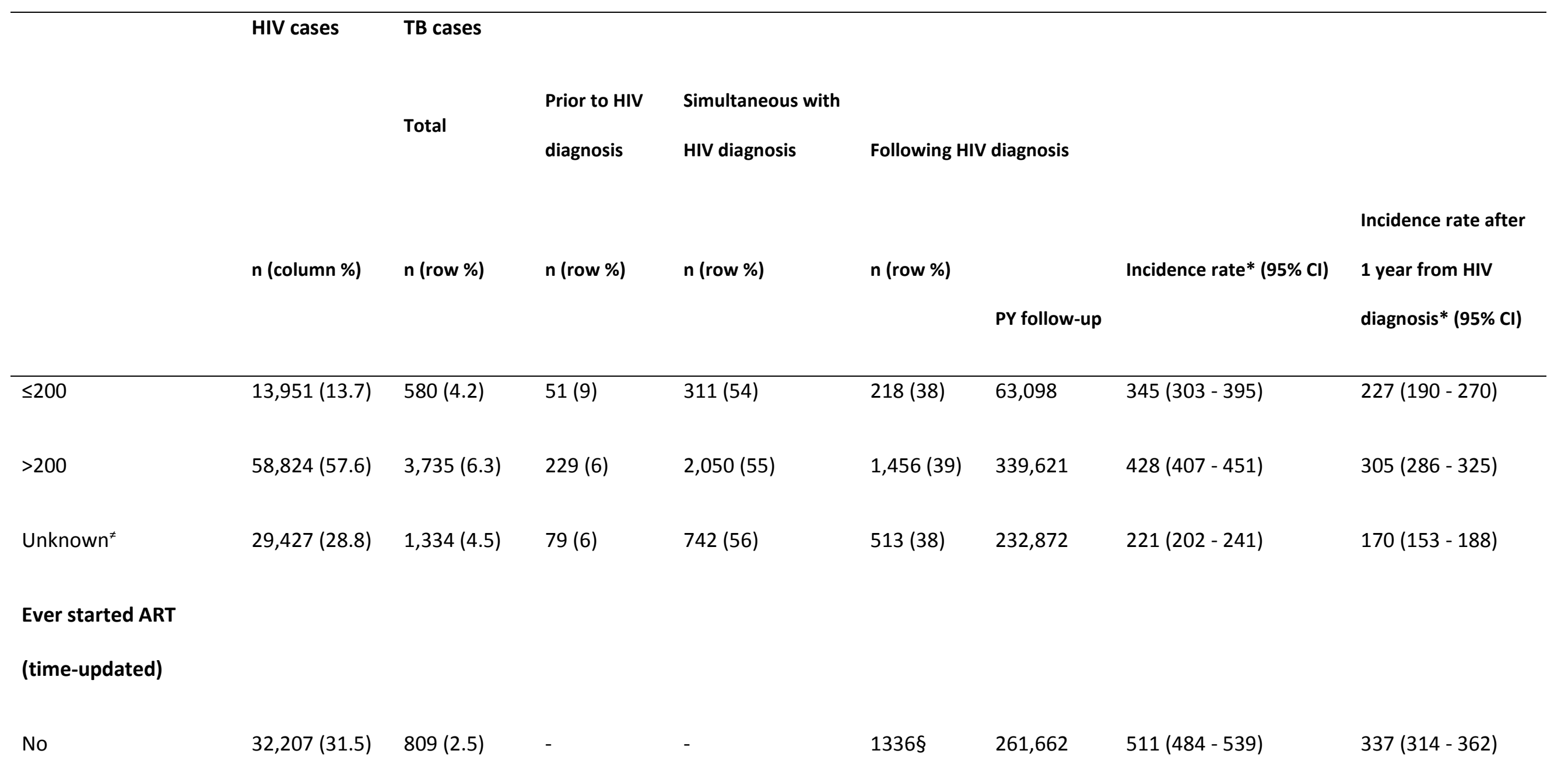




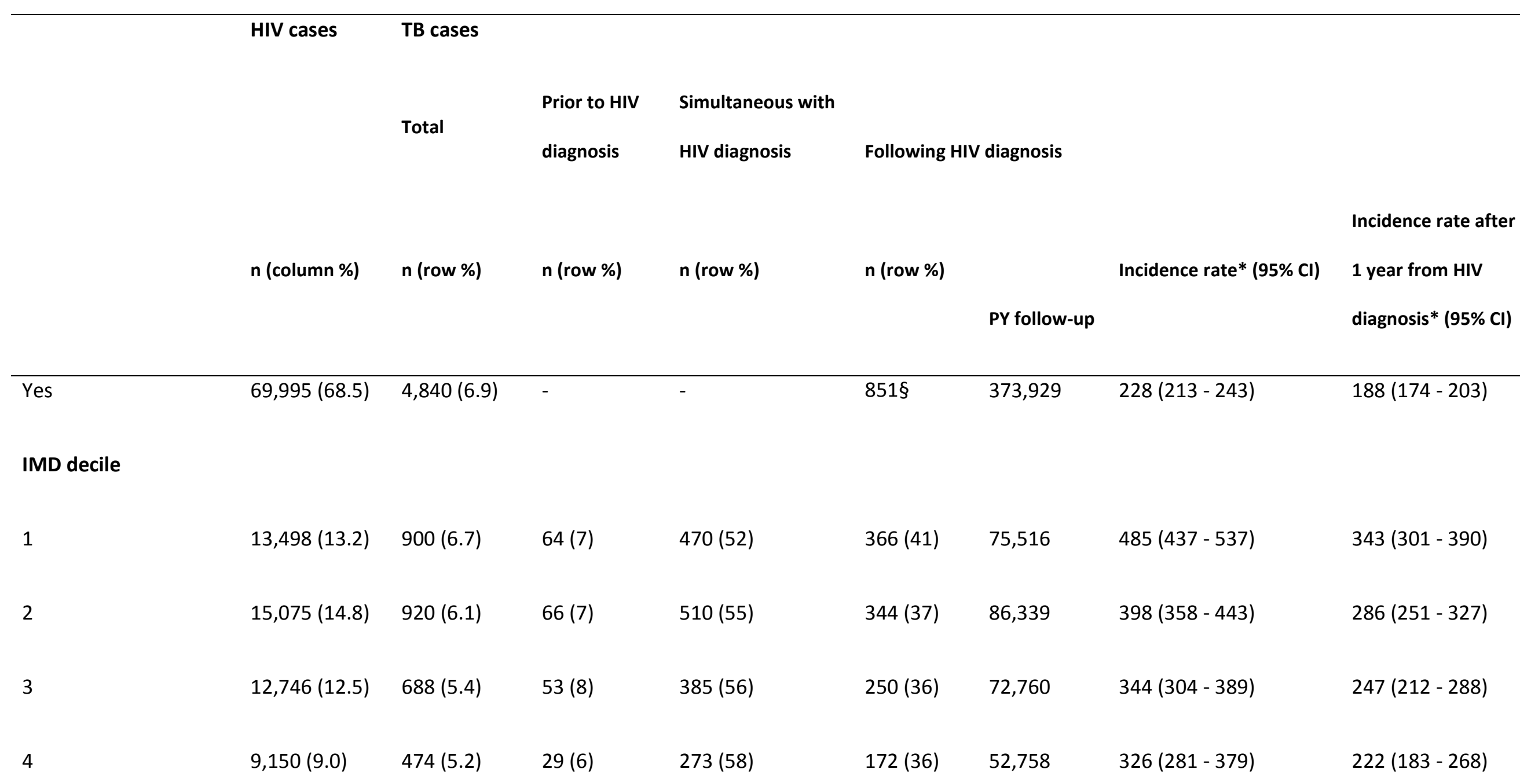




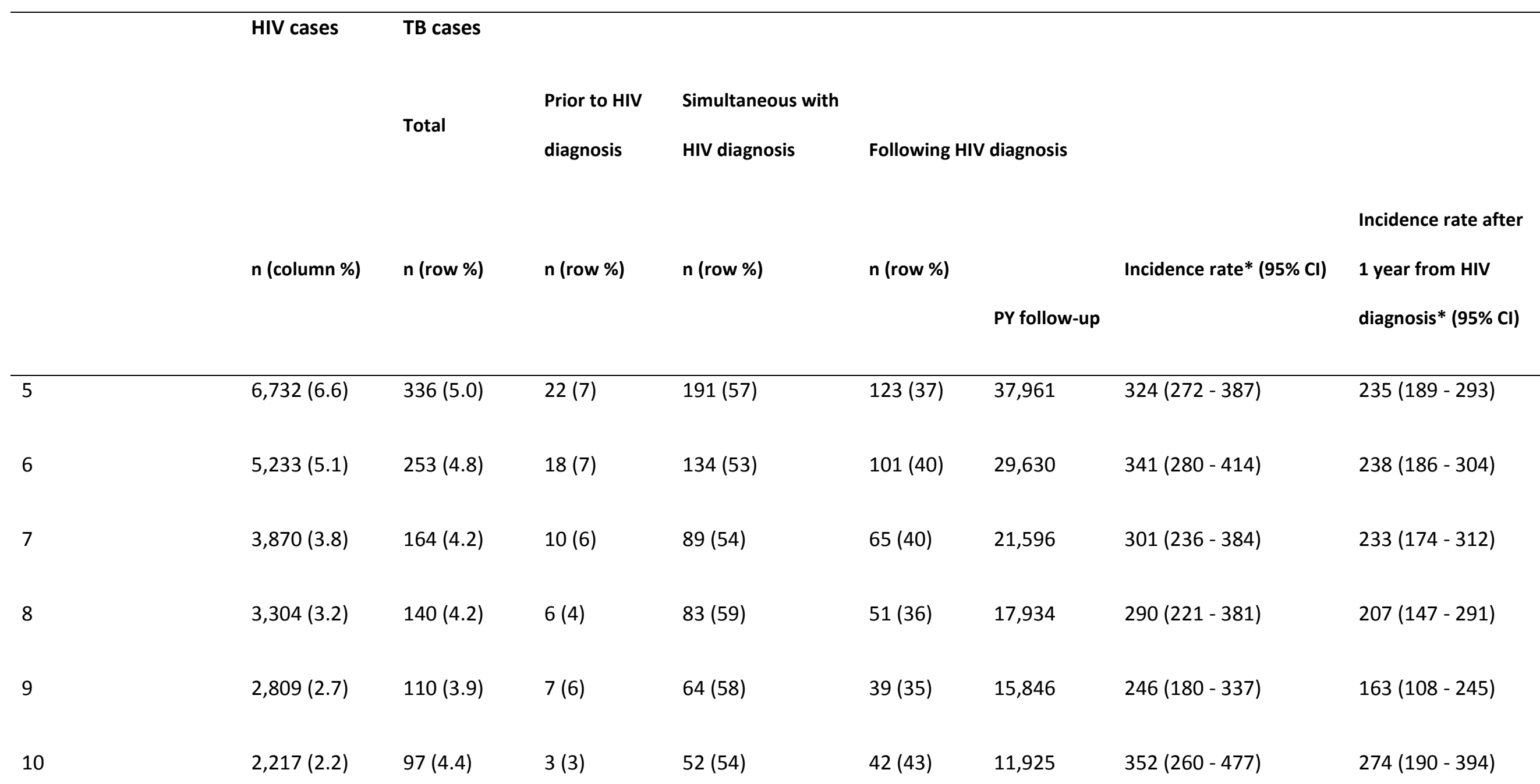




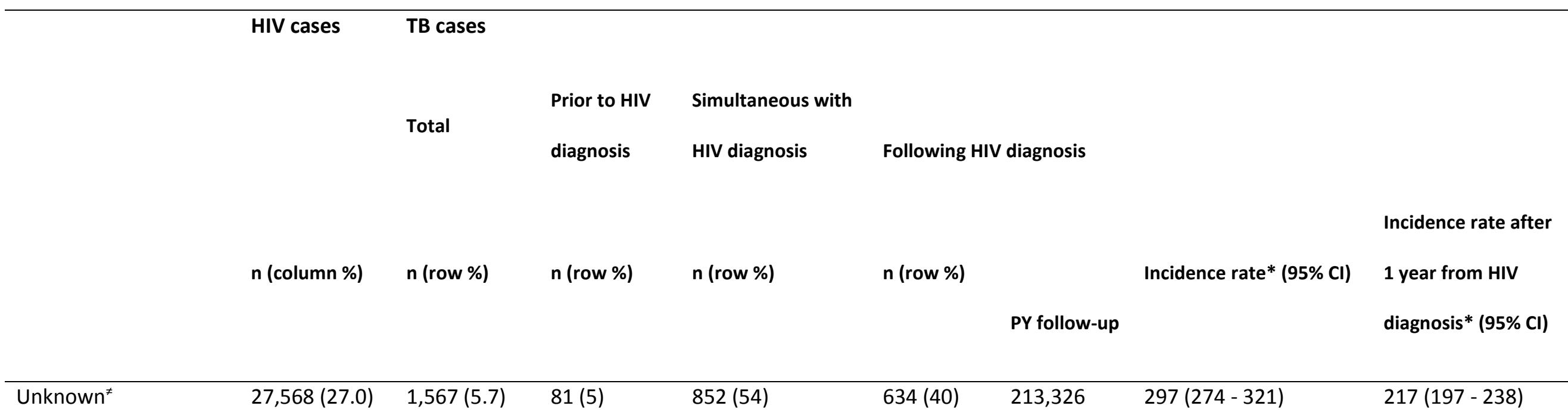

* Incidence is given per 100,000 population aged $\geq 15$ years, per year. + Incidence rates are calculated for time-updated CD4 count. ${ }^{*}$ Unknown strata includes both 

diagnosis) among PLHIV in England, Wales and Northern Ireland from 2000 to 2014

\begin{tabular}{lllll}
\hline & & & Univariable & Multivariable \\
& TB cases & PY & IRR (95\% CI) & IRR (95\% CI) \\
\hline Route of HIV infection & & & & \\
MSM & 184 & 172,708 & $1.00(P<0.001)$ & $1.00(P<0.001)$ \\
Male heterosexual & 474 & 82,460 & $5.40(4.55-6.40)$ & $1.70(1.38-2.10)$ \\
Female heterosexual & 837 & 148,391 & $5.29(4.51-6.21)$ & $1.86(1.51-2.29)$ \\
Male PWID & 61 & 5,895 & $9.71(7.27-12.97)$ & $5.47(4.07-7.35)$ \\
Female PWID & 16 & 2,514 & $5.97(3.58-9.95)$ & $4.59(2.75-7.67)$ \\
Blood/Tissue transfer & 14 & 2,251 & $5.84(3.39-10.05)$ & $2.70(1.55-4.71)$
\end{tabular}


Mother-to-child

\section{Ethnicity/Country of birth}

White, UK-born

Black African, UK-born

Other ethnicity, UK-born

Ethnicity unknown, UK-born

Born in low-TB incidence country

White, born in high-TB incidence

country

Black African, born in high-TB

incidence country

Other ethnicity, born in high-TB
494

$9.51(3.91-23.11) \quad 2.80(1.13-6.97)$

134

13

4,317

$2.86(1.62-5.06)$

$1.97(1.10-3.51)$

31

12,040

252

53,647

38

12,606

1,093

105
148,017

2,219
$1.92(1.29-2.84)$

$33(1.02-1.73)$

$2.87(2.00-4.11) \quad 2.19(1.53-3.15)$

$7.02(5.87-8.40) \quad 4.27(3.42-5.33)$

$4.50(3.48-5.80) \quad 3.36(2.57-4.39)$
$2.45(1.66-3.62)$

[Type text] 
incidence country

Ethnicity unknown, born in high-

$2.95(0.41-21.07) \quad 1.35(0.19-9.71)$

TB incidence country

White, country of birth unknown

15,491

$0.74(0.41-1.33) \quad 0.52(0.29-0.94)$

Other ethnicity, country of birth

unknown

\section{CD4 count}

$\geq 500$

259

185,719

$1.00(P<0.001)$

$350-499$

293

113,185

$1.86(1.57-2.19)$

200-349

427

80,443

$3.81(3.26-4.44)$

100-199

332

24,367

9.77 (8.30 - 11.49)

$50-99$

137

6,093

$16.12(13.11-$ 
19.83)

$0-49$

\section{Ever on ART}

No

Yes

Viral load at diagnosis

$\leq 200$

$>200$

Age at HIV diagnosis

$15-24$

169

928

663

154
1,063

261,249

$1.15(0.97-1.36)$

25.64)

07,477 $\quad 1.00(P<0.001) \quad *$

$307,237 \quad 0.25(0.23-0.28)$

$\begin{array}{ll}3,347 & 1.00(P=0.006) \\ 61,249 & 1.15(0.97-1.36)\end{array}$

$0.95(0.79-1.13) \quad 0.92(0.77-1.10)$ 


$\begin{array}{lcccc}25-34 & 714 & 170,957 & 1.14(1.02-1.28) & 1.06(0.94-1.19) \\ 35-44 & 477 & 130,441 & 1.00(P<0.001) & 1.00(P=0.332) \\ 45-64 & 220 & 61,028 & 0.99(0.84-1.16) & 1.11(0.95-1.31) \\ \geq 65 & 11 & 3,484 & 0.86(0.47-1.57) & 0.92(0.51-1.68)\end{array}$

\section{Year of HIV diagnosis}

(for each year increase from

$$
1,591 \quad 414,714 \quad 0.98(0.97-1.00) \quad 1.02(1.00-1.04)
$$

2000)

$P=0.036 \quad P=0.014$

\section{IMD decile (England and Wales}

only)

1

264

51,685

$1.00(P<0.001)$

2

269

63,391

$0.83(0.70-0.98)$

[Type text] 
4

5

6

7

8

9

10

486

[Type text]
$193 \quad 54,955 \quad 0.69(0.57-0.83)$

$127 \quad 38,159 \quad 0.65(0.53-0.81)$

$83 \quad 26,725 \quad 0.61(0.48-0.78)$

$78 \quad 20,986 \quad 0.73(0.57-0.94)$

$47 \quad 15,254 \quad 0.60(0.44-0.82)$

$38 \quad 12,644 \quad 0.59(0.42-0.83)$

$24 \quad 10,743 \quad 0.44(0.29-0.66)$

$32 \quad 8,326 \quad 0.75(0.52-1.09)$ 

count or age at HIV diagnosis. Viral load was not included in the multivariable model due to collinearity with CD4 count and ART status. *Interaction present between time-updated CD4 count and time-updated ART status, see Table 4 and Table 3. +Not calculated as numerator was zero. ART: anti-retroviral therapy, Cl: confidence 
492

493 


\section{CD4 count (cells/ $\mu$ l)}

\begin{tabular}{lllllll} 
& $\geq 500$ & $350-499$ & $200-349$ & $100-199$ & $50-99$ & $0-49$ \\
Ever on ART & IRR $(95 \%$ Cl) & IRR (95\% CI) & IRR (95\% CI) & IRR (95\% CI) & IRR (95\% CI) & IRR (95\% CI) \\
\hline No & 1.00 & 1.00 & 1.00 & 1.00 & 1.00 & 1.00 \\
Yes & $0.07(0.05-0.10)$ & $0.14(0.11-0.18)$ & $0.21(0.17-0.25)$ & $0.32(0.26-0.40)$ & $0.35(0.25-0.49)$ & $0.49(0.35-0.69)$
\end{tabular}

Incidence rate ratios derived from multivariable Poisson regression of the association between time-updated ART status and TB disease, stratified by CD4 count. Model

adjusted for the variables in the multivariable model in Table 2. 62,684 PLHIV were included in this analysis; 32,319 were excluded from the model due to missing data 
501

502

503

\begin{tabular}{|c|c|c|}
\hline \multirow[b]{3}{*}{ CD4 count (cells/ $\mu l)$} & \multicolumn{2}{|l|}{ Ever on ART } \\
\hline & No & Yes \\
\hline & IRR (95\% CI) & IRR $(95 \% \mathrm{Cl})$ \\
\hline$\geq 500$ & $1 \cdot 00$ & 1.00 \\
\hline $350-499$ & $1.28(1.06-1.55)$ & $2 \cdot 51(1.77-3.56)$ \\
\hline $200-349$ & $2.22(1.84-2.66)$ & $6.37(4.66-8.72)$ \\
\hline 100-199 & $4.74(3.79-5.93)$ & $21 \cdot 21(15 \cdot 59-28 \cdot 85)$ \\
\hline $50-99$ & $7 \cdot 07(5 \cdot 26-9 \cdot 51)$ & $34 \cdot 29(24 \cdot 10-48 \cdot 77)$ \\
\hline $0-49$ & $6.42(4.87-8.46)$ & $44 \cdot 21(30 \cdot 90-63 \cdot 24)$ \\
\hline
\end{tabular}

Ever on ART
504
Table 4: Multivariable Poisson regression of the association between time-updated CD4 count and TB disease, stratified by ART status, among PLHIV in England, Wales and Northern Ireland from 2000 to 2014
Incidence rate ratios derived from multivariable Poisson regression of the association between timeupdated CD4 count and TB disease, stratified by ART status. 62,684 PLHIV were included in this analysis; 32,319 were excluded from the model due to missing data on ethnicity and country of birth, route of HIV infection, CD4 count or age at HIV diagnosis. Model adjusted for the variables in the multivariable model in Table 2. ART: anti-retroviral therapy, Cl: confidence interval, IRR: incidence rate ratio, TB: tuberculosis.

[Type text] 